\title{
Simple Instruments in Radio Astronomy
}

\author{
Nguyen Quang Rieu \\ Observatoire de Paris, Departement DEMIRM, 61 Avenue de \\ l'Observatoire, 75014 Paris, France. e-mail: \\ Nguyen-Quang.Rieu@obspm.fr
}

\begin{abstract}
Radio astronomy has a major role in the study of the universe. The spiral structure of our Galaxy and the cosmic background radiation were first detected, and the dense component of interstellar gas is studied, at radio wavelengths. COBE revealed very weak temperature fluctuations in the microwave background, considered to be the seeds of galaxies and clusters of galaxies. Most electromagnetic radiation from outer space is absorbed or reflected by the Earth's atmosphere, except in two narrow spectral windows: the visible-near-infrared and the radio, which are nearly transparent. Centimetre and longer radio waves propagate almost freely in space; observations of them are practically independent of weather. Turbulence in our atmosphere does not distort the wavefront, which simplifies the building of radio telescopes, because no devices are needed to correct for it. Observations at these wavelengths can be made in high atmospheric humidity, or where the sky is not clear enough for optical telescopes.

Simple instruments operating at radio wavelengths can be built at low cost in tropical countries, to teach students and to familiarize them with radio astronomy. We describe a two-antennae radio interferometer and a single-dish radio telescope operating at centimetre wavelengths. The Sun and strong synchrotron radio-sources, like Cassiopeia $\mathrm{A}$ and Cygnus A, are potential targets.
\end{abstract}

\section{Introduction}

Thousands of bright stars in the Milky Way can be seen with the naked eye. Light from remote galaxies is detected with large optical telescopes. Astronomical objects emit not only in the visible but also in the whole electromagnetic spectrum. The investigation of the physical conditions of astronomical objects requires observations in as many wavebands as possible, from gamma and $\mathrm{X}$ rays through the ultraviolet and the visible to infrared and radio waves. The frequency and the spectral extent of the cosmic radiation depend on the radiation mechanisms and on the physical conditions, in particular the temperature, the density and the magnetic field in the object. Radiation from outer space is, 
however, absorbed or reflected by the Earth's atmosphere in a large part of the electromagnetic spectrum. There exist only two narrow spectral windows which are almost transparent to cosmic radiation, namely the visible-near infrared and the radio windows. Outside these windows, the observations must be made by telescopes on board balloons or from satellites launched above the terrestrial atmosphere.

\section{Some Salient Results in Radio Astronomy}

Radio-astronomical observations play a major role in the investigation of the universe. The frequency band observable with ground-based radio telescopes ranges from a few Megahertz to 800 Gigahertz. The cosmic background radiation was discovered at radio wavelengths (Penzias and Wilson, 1965). Its spectrum can be fit accurately by a $2.735 \mathrm{~K}$ black body curve peaking in the millimetre spectral region. The observations with the satellite COBE (Smoot et al. 1991) and balloon-borne experiments (de Bernardis et al. 2000; Hanany et al. 2000) have revealed that the background radiation exhibits tiny temperature fluctuations which are the imprint of the large scale structures in the primordial universe. These temperature anisotropies contain basic information allowing astronomers to discriminate between cosmological models.

The spiral structure of the Milky Way was first discovered by the extensive mapping using the 21-cm line of atomic hydrogen, which is also used to map the grand-design spiral pattern of other galaxies (e.g. van de Hulst et al., 1954). Figure 1 shows the image of the spiral galaxy NGC 6946 observed in the 21-cm line.

Furthermore, observations of the 21-cm line have shown that the rotation curves of several galaxies are much flatter beyond the optical disks than predicted by Kepler's law, suggesting that some dark matter may exist in the halos of galaxies (e.g. Sancisi and van Albada, 1987). All these radio data should provide valuable constraints on a number of cosmological parameters.

Gas in dense dark clouds is essentially in molecular form, thereby precluding observations in the visible, namely the $\mathrm{H} \alpha$ line, and in the 21-cm line of atomic hydrogen. The investigation of this dense gas component has been made possible thanks to the radio observations of interstellar molecules. The envelopes of evolved stars and the remnants of supernovae are rich in molecules, which are reprocessed within the stars and injected into the interstellar medium. Molecular-line observations are, therefore, useful for studying the chemical evolution of our Galaxy. Furthermore, spectacular maser action was discovered in molecular clouds capable of amplifying the background radiation by several orders of magnitude.

Observations of highly redshifted radiation from remote galaxies and quasars are becoming a favourite field of research for instruments operating at long radio wavelengths. Since long radio waves propagate almost freely in space, radio observations do not depend strongly on the weather conditions. The presence of turbulence in the Earth's atmosphere does not distort the wavefront of radio signals from outer space. This fact simplifies the building of radio telescopes which do not require sophisticated devices to correct the turbulence effect. 


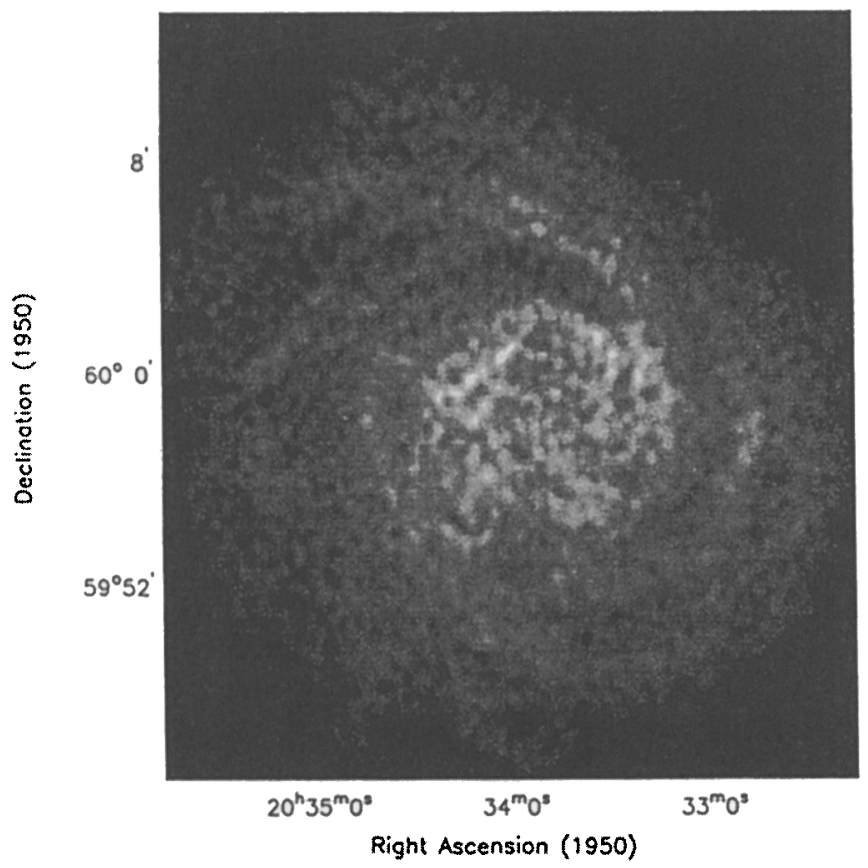

Figure 1. The spiral structure of NGC 6946 observed in the hydrogen line at $\lambda=21 \mathrm{~cm}$ with the Westerbork Supersynthesis Radio Telescope in the Netherlands (Boulanger and Viallefond, 1992).

\section{Instruments in Radio Astronomy}

An astronomical instrument is characterized by its sensitivity and its resolving power. It should have a large collecting area to achieve this double performance. The number of photons collected by the instrument increases with its size. The angular resolution is $\alpha \sim \lambda / D, \lambda$ is the observing wavelength and $D$ the diameter of the instrument. In order to obtain angular resolutions similar to those achieved in optical astronomy, radio astronomers have to build antennae whose sizes are larger than those of optical telescopes by a factor of $\lambda_{\text {radio }} / \lambda_{\text {optical }}$. The resolution of a $10-\mathrm{m}$ optical telescope built with advanced technology working at $\lambda_{\text {optical }} \sim 0.6 \mu \mathrm{m}$ is theoretically $\sim 0.015$ arcsec. The diameter of a radio telescope working at $\lambda_{\text {radio }} \sim 10 \mathrm{~cm}$ with the same resolution would be $\sim 1670 \mathrm{~km}$. Building such a huge single antenna is beyond the range of present technology. But radio interferometers consisting of a network of small antennae separated from each other by thousands of kilometers are currently used in radio astronomy to achieve high resolution.

Astronomical observations at centimetre wavelengths or at longer wavelengths are quite appropriate for countries where the humidity of the atmosphere is significant and the sky is not sufficiently clear to use optical telescopes. This is the choice of Indian scientists who built their Giant Metrewave Radio Telescope 
(GMRT) near Pune. This instrument consists of 30 parabolic antennae, each of 45-m diameter, functioning as an interferometer in the form of the letter ' $\mathrm{Y}$ '. Each arm extends to $\sim 15 \mathrm{~km}$ from the centre of the system. With a collecting area of $\sim 30,000$ square metres, the GMRT is the largest interferometric array in the world. The GMRT which operates at low radio frequencies, from $150 \mathrm{MHz}$ $(\lambda=2 \mathrm{~m})$ to $1420 \mathrm{MHz}(\lambda=21 \mathrm{~cm})$, is devoted especially to the detection of embryonic galaxies present in the early universe, through the $21-\mathrm{cm}$ hydrogen line expected to be redshifted to metre wavelengths because of the expansion of the universe. The angular resolution of the GMRT is as high as $\sim 2 \operatorname{arcsec}$ at $\lambda$ $=21 \mathrm{~cm}$.

Great progress has also been made in the development of detectors, by the use of superconductor material. All the receiving system is cooled to a very low temperature, $\sim 4 \mathrm{~K}$ by liquid helium in order to minimize the receiver noise.

In countries where the research in astronomy is not yet well-developed, equipments at low cost are appropriate. A simple instrument can be used by teachers to train students in physics and to familiarize them with radio astronomy by considering the universe as a laboratory. The radio emission from the closest star, the Sun, can be easily detected. Strong synchrotron radio sources, like Cassiopeia A and Cygnus A can also be possible targets. A small instrument equipped with an uncooled receiver is most suitable for this purpose.

\section{The Basic Concept of Interferometry}

Let us consider the one-dimensional case with two antennae $A_{1}$ and $A_{2}$ separated by a distance $L$ (Figure 2). The radio signal of frequency $\nu$ coming from a point source is received at $A_{1}$. The output voltage is $U_{1}=E \sin (2 \pi \nu t)$. The signal reaches antenna $A_{2}$ with a time delay $\tau=(L \sin \theta) / c$, resulting in a phase shift $\phi=2 \pi \nu \tau ; c$ is the velocity of light and $\theta$ denotes the direction of the incoming signal, that is the angle between the direction of the radio source with the perpendicular to the baseline.

The output voltage at antenna 2 is then, $U_{2}=E \sin (2 \pi \nu t+\phi)$. If we now add the two signals by connecting the two antennae to the same receiver, we get the interferometric signal $S$ :

$$
S=U_{1}+U_{2}=E \sin (2 \pi \nu t)+E \sin (2 \pi \nu t+\phi)
$$

This expression is equivalent to:

$$
S=2 E \cos (\phi / 2) \sin (2 \pi \nu t+\phi / 2)
$$

After detection, we get an intensity $I$ proportional to the square of the "interferometric term" $\cos (\phi / 2)$, i.e.:

$$
I=S^{2}=4 E^{2} \cos ^{2}(\phi / 2)=2 E^{2}(1+\cos \phi)
$$

with $\phi=(2 \pi L \sin \theta) / \lambda$ 


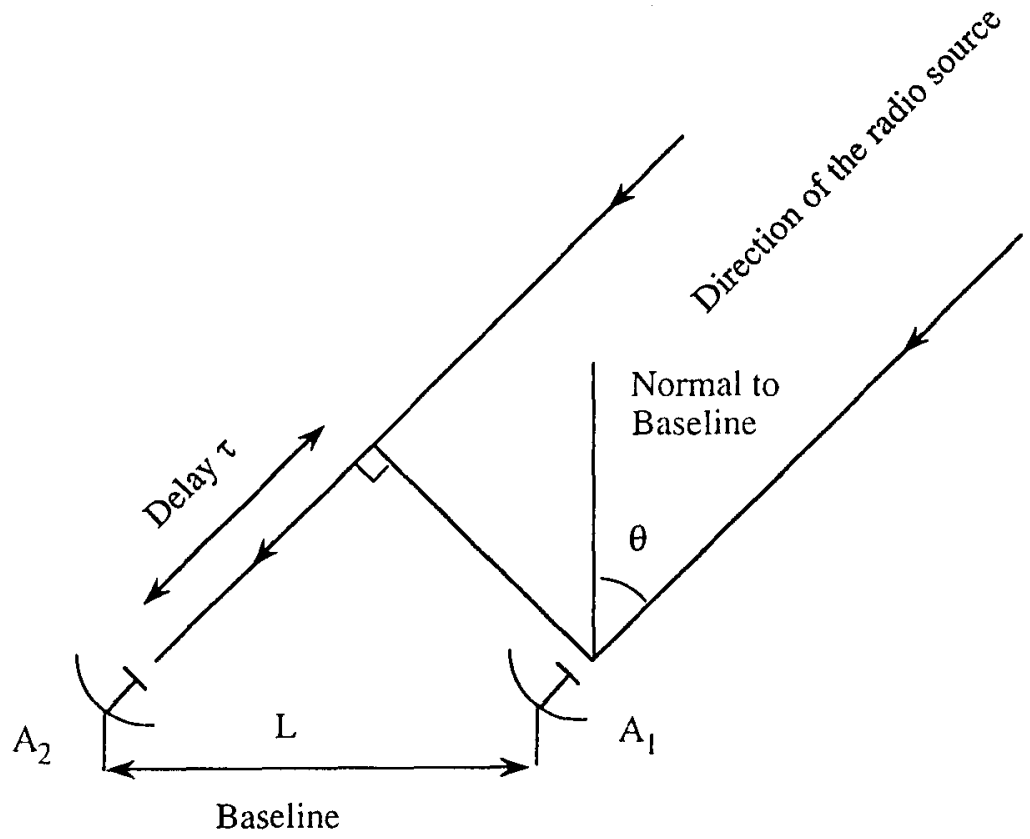

Figure 2. A two-antennae interferometer

As the radio source, $S$, is rising towards the zenith, $\theta$ varies, as well as $\phi$, as a function of time. For a baseline oriented east-west, it can be shown that (by using formulae of spherical trigonometry in the spherical triangle PSE):

$$
\sin \theta=\cos \delta \sinh
$$

where $\delta$ is the declination of the radio source and $h$ is the hour angle.

Eqs. 1 and 2 show that the intensity $I$ of the signal of the source varies periodically with maxima occurring at hour angles $h$ such that:

$$
\sinh =n \lambda /(L \cos \delta)
$$

where $n$ is an integer, varying from 0 to $n$.

From Eq.3 we derive the time interval between the two nearest maxima:

$$
\Delta h=\lambda /(L \cos \delta \cos h)
$$

Figure 4 shows the fringe pattern calculated around $h=0$, with $\lambda=0.5 \mathrm{~m}$, $L=25 \mathrm{~m}$ and $\delta=15^{\circ}$ (Thai-Q-Tung, 1997). 


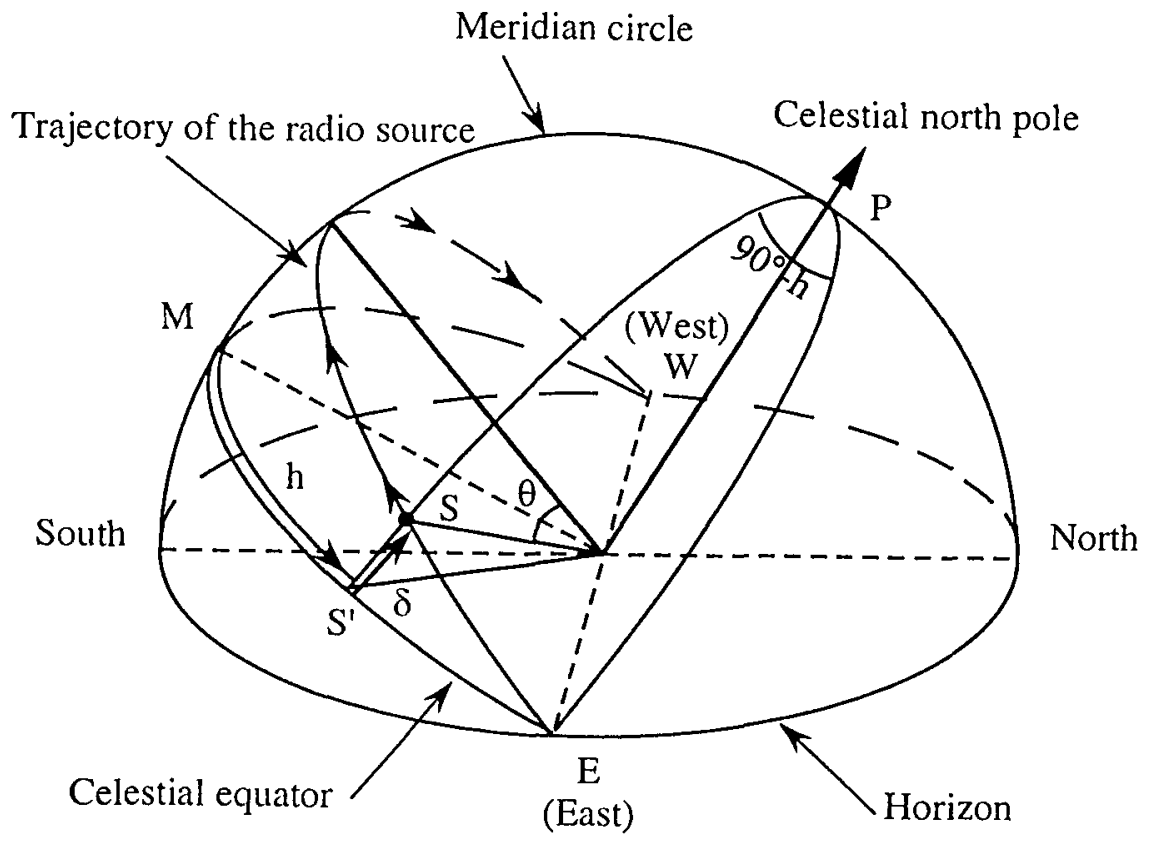

Figure 3. Observation of a radiosource

\section{Extended Sources}

Equation (1) can be generalized to calculate the response of an extended (one dimensional) source whose size is $D$ (Thai-Q-Tung, 1997). The intensity of the signal is:

$$
I=2 \int_{-D / 2}^{D / 2} E_{x}^{2}[(1+\cos (2 \pi L \sin (\theta+x) / \lambda)] d x
$$

For the sake of simplicity, we assume that $x$ is small and the intensity distribution is uniform and symmetrical with respect to the $x$ axis. We then get:

$$
I=2 E_{0}^{2} D+2 E_{0}^{2} \cos (2 \pi L \sin \theta / \lambda) \sin (\pi L D \cos \theta / \lambda) /(\pi L \cos \theta / \lambda)
$$

Figure 4 shows the fringe pattern (dotted lines). The fringe amplitude $\mathrm{V}(\mathrm{u})$ now changes with $\theta$ : 


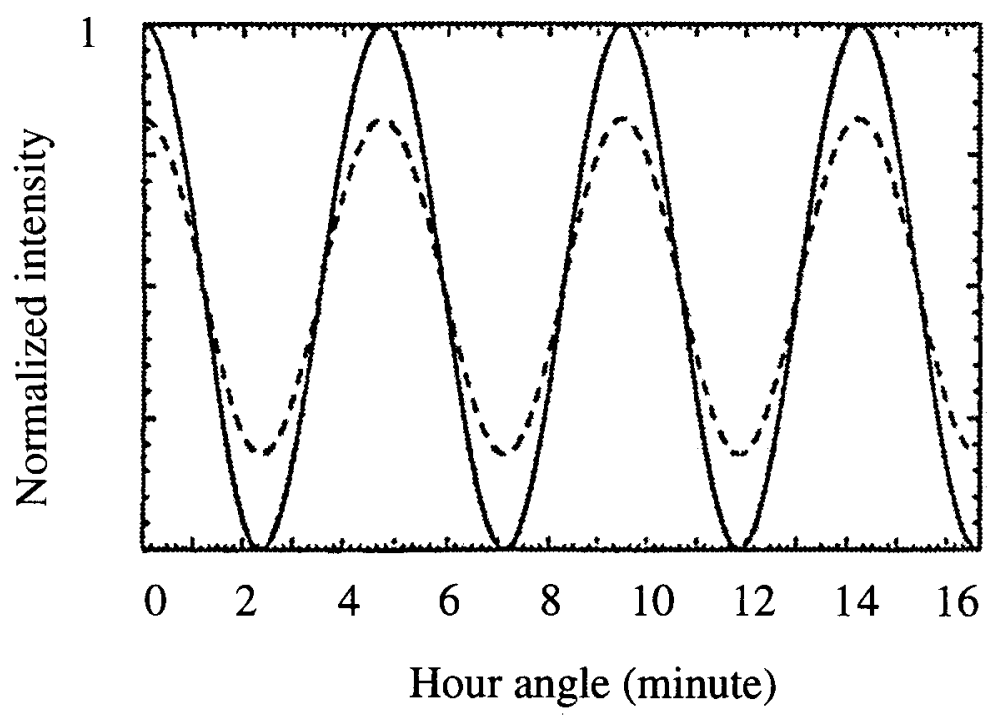

Figure 4. Fringe pattern: point source (full line); uniform extended source (dotted line).

$$
V(u)=2 E_{0}^{2} \sin (\pi D u) / \pi u
$$

with the spatial frequency $u=L \cos \theta / \lambda$

The brightness distribution is restored by Fourier transform:

$$
B(x)=\int_{-\infty}^{\infty} V(u) \exp (-i 2 \pi u x) d u
$$

The restoration of the brightness using Eq.5 is illustrated in Figure 5.

\section{A Two-Element Radio Interferometer}

In the following we describe a simple radio interferometer. The two antennae are ordinary TV dipole Yagi aerials fixed to two flagpoles. The radio signals coming from the antennae are added and sent to a video-recorder which is used as a receiver. After detection, the radio signal is sent to an analog-digital converter and then to a computer which records the signal and displays the fringe pattern (Biraud, 1985; Biraud and Darchy, 1990).

High-gain antennae (gain $\geq 10 \mathrm{~dB}$ ) are required to obtain a large equivalent collecting area. A gain of $16 \mathrm{~dB}$ corresponds to a peak directivity of the antenna main beam $\mathrm{G}=40$. The collecting area is $A=\lambda^{2} G /(4 \pi)$ and the main beam is $\alpha=1.2 \lambda / D$, with an antenna size $D=2 \sqrt{A / \pi}$. At $\lambda=0.5 \mathrm{~m}$, one finds 


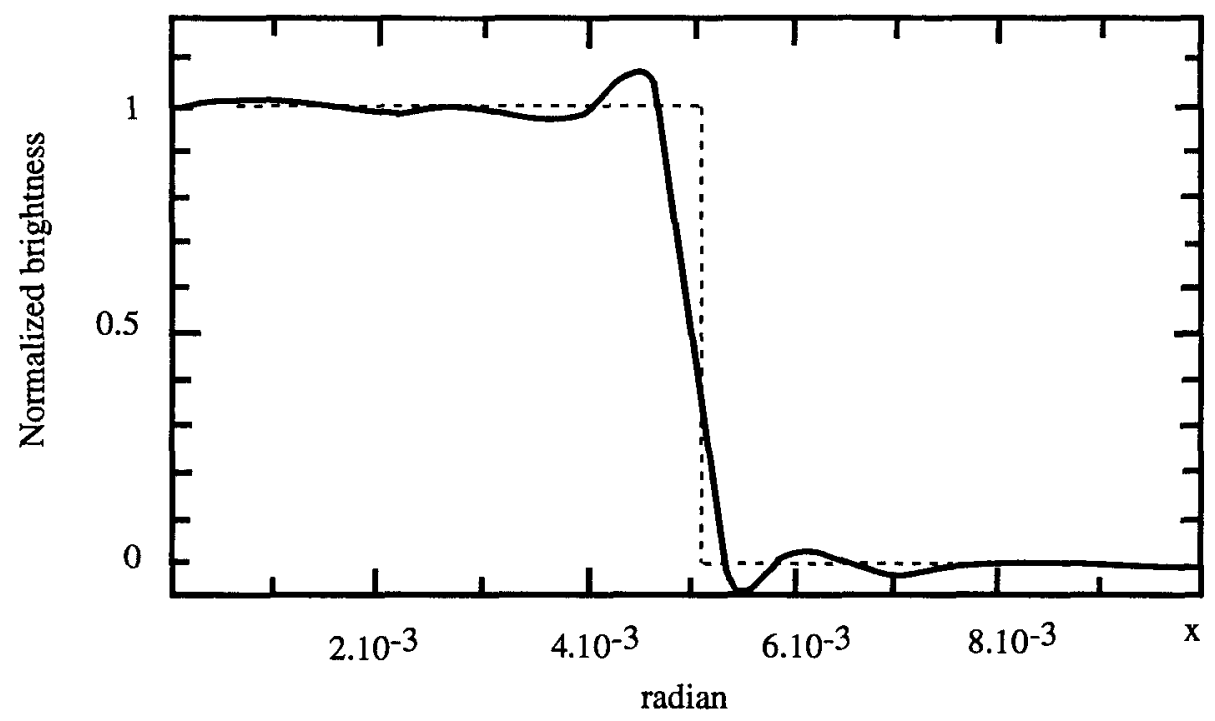

Figure 5. Signal restoration : model (dotted line), restored brightness (full line).

$A=0.8 \mathrm{~m}^{2}$ and $\alpha=34^{\circ}$. The beam is large enough to maintain the fringe amplitude of the radio source practically constant during several hours, facilitating the pointing procedure. The pointing of the antennae can be made manually. A baseline $L \sim 25 \mathrm{~m}$ gives a fringe interval (interval between two nearest maxima) of $\sim 1.5^{\circ}$. This value, much larger than the solar diameter $(\sim 32 \operatorname{arcmin})$, is suitable to detect fringes from the Sun. Such an instrument with an east-west baseline $L=25 \mathrm{~m}$ was used to observe the Sun during the solar eclipse in Phan Thiet (Vietnam) on October 24, 1995 (Figure 6).

Figure 7 shows the fringe pattern in different phases of the eclipse. The fringe amplitude decreases notably when the Sun was totally hidden, between $11 \mathrm{~h} 14 \mathrm{~m}$ and $11 \mathrm{~h} 16 \mathrm{~m}$ (local time). The Sun which had a declination $\delta=-11.5^{\circ}$, culminated at $11 \mathrm{~h} 32 \mathrm{~m}$.

Observation started at $09 \mathrm{~h} 10 \mathrm{~m}$ (hour angle $h=-2 \mathrm{~h} 22 \mathrm{~m}$ ) and stopped at $12 \mathrm{~h} 00 \mathrm{~m}(h=+0 \mathrm{~h} 28 \mathrm{~m})$. The fringe interval is $5 \mathrm{~m} 45 \mathrm{~s}$ at the beginning and $4 \mathrm{~m} 43 \mathrm{~s}$ at the end of the observation (Eq.4).

The observations suffered from the interference due to the presence of many eclipse watchers riding motorcycles and driving cars. Furthermore, a station for radio telephones was installed near the observation site. The record represented in Figure 7 results from a cleaning procedure to eliminate the interference peaks.

The solar flux $F$ is on average $\sim 2.10^{-21} \mathrm{Wm}^{-2} \mathrm{~Hz}^{-1}$, corresponding to a peak antenna temperature $T_{a} \sim 45 \mathrm{~K}$, at $\lambda=0.5 \mathrm{~m}$. The antenna is electrically equivalent to a resistor. The antenna temperature is the temperature of the antenna radiation resistance, which depends on the temperature of the emitting region in the sky. Figure 7 shows that the signal-to-noise ratio is excellent. The fringe amplitude decreases when the baseline increases. The fringes eventually disappear when the baseline becomes too large. The baseline of the interferom- 


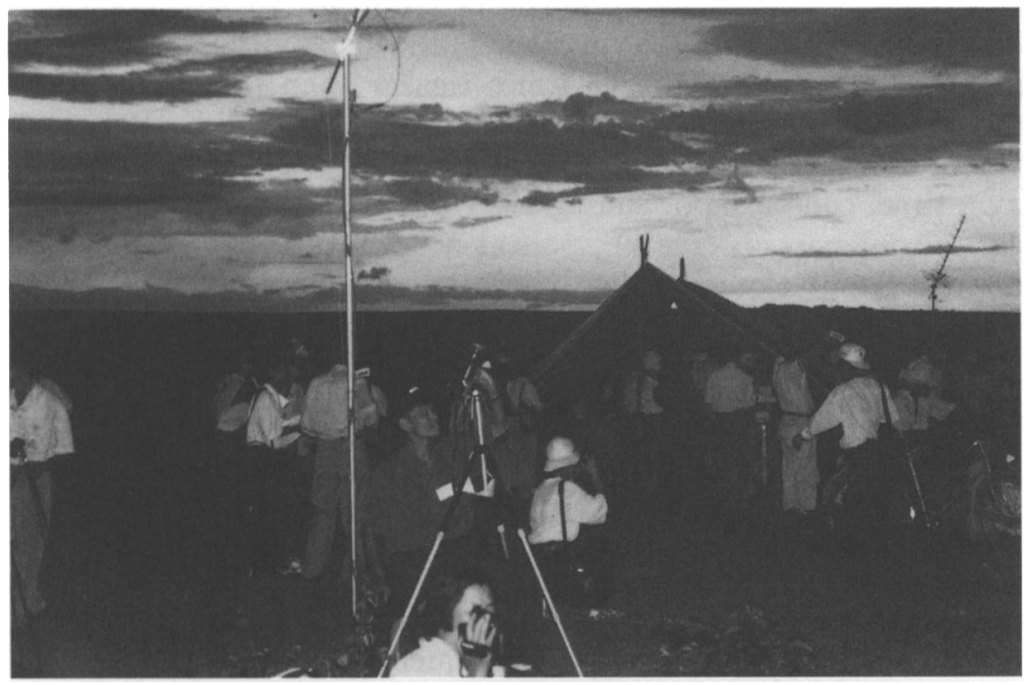

Figure 6. The two antennae of the interferometer (visible in the foreground and background) used to observe the solar eclipse in Phan Thiet (Vietnam).

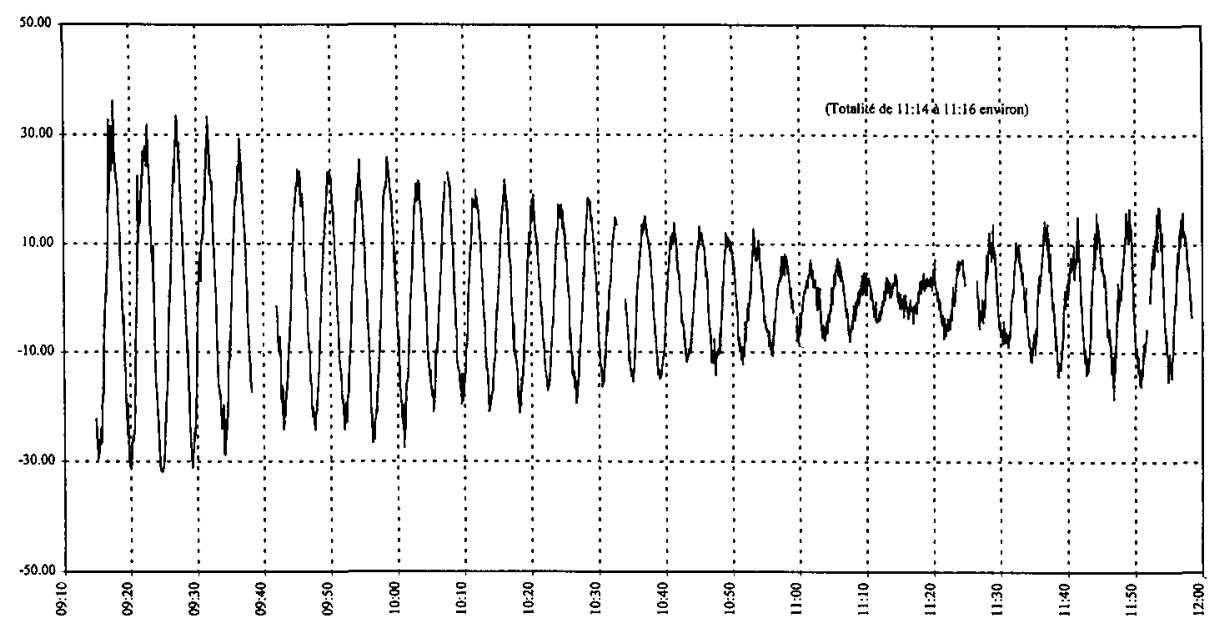

Figure 7. Fringe pattern during the different phases of the solar eclipse in Phan Thiet, Vietnam. B. Darchy, Observatoire de Paris 
eter can be varied and oriented in different directions to measure the diameter or to map the emission of the radio source (Nguyen-Q-Rieu, 1993).It is possible to observe a few synchrotron radio sources such as the supernova remnant Cassiopeia A. This source is $\sim 45$ times weaker than the Sun at $\lambda=0.5 \mathrm{~m}$. The antenna temperature of Cassiopeia A would be $\sim 1 \mathrm{~K}$. It is possible to detect this source and strong radio galaxies like Cygnus $\mathrm{A}$, if we allow for a long integration time, consisting of adding several tens of fringes together.

Solar astronomers observe sunspots at $\lambda \sim 10 \mathrm{~cm}$ to estimate the activity of the Sun. A radio interferometer with an east-west baseline of $350 \mathrm{~m}$ consisting of two 5-m dishes, gives an angular resolution $\sim 1$ arcminute, which is adequate for the purpose. With such an instrument which has a primary beam (field of view) $\sim 1^{\circ}$, we can detect sunspots whose fluxes are $10^{-22}-10^{-21} \mathrm{Wm}^{-2} \mathrm{~Hz}^{-1}$.

\section{A Single-Dish Radio Telescope}

A simple single dish radio telescope can be built with an 0.7-m parabolic antenna designed to receive television signals from satellites (Darchy and Flouret, 1999). This type of antenna operates around frequencies of $11 \mathrm{GHz}(\lambda=0.027 \mathrm{~m})$. The antenna beam, $\alpha_{b} \sim 2.6^{\circ}$, is narrow enough to require a pointing accuracy which should be better than $1^{\circ}$. For the Sun, its optical image formed at the antenna focus can be used to check the pointing. The radio emission profile appears approximately as a gaussian curve, while the Sun drifts along the beam as the result of the diurnal and solar proper motions. At $\lambda=0.027 \mathrm{~m}$, the observed antenna temperature $T_{a}$ of the Sun is $\sim 360 \mathrm{~K}$. The solar brightness temperature, $T_{b} \approx T_{a}\left(\alpha_{b} / \alpha_{S u n}\right)^{2}$, is $8500 \mathrm{~K}$, if the angular diameter of the Sun $\alpha_{\text {Sun }}$ is $32^{\circ}$.

The flux $F$ of the synchrotron emission from radio sources decreases at short wavelengths, according to the power law $F \propto \lambda^{\beta}$. For Cas $\mathrm{A}$, the spectral index $\beta$ is 0.77 . The flux of Cas $\mathrm{A}$ at $\lambda=0.027 \mathrm{~m}$ is $\sim 4.610^{-24} \mathrm{Wm}^{-2} \mathrm{~Hz}^{-1}$. The collecting area of a $0.7-\mathrm{m}$ parabola is $A=0.38 \mathrm{~m}^{2}$. The antenna temperature is given by $T_{a}=F \times A /(2 k)$, where $k$ is the Boltzmann constant. At $\lambda=0.027 \mathrm{~m}$, the antenna temperature of Cas $A$ is only $T_{a} \sim 0.06 \mathrm{~K}$, which is $\sim 6000$ times weaker than that of the Sun. Furthermore, at $\lambda=0.027 \mathrm{~m}$, the observation is rather sensitive to the weather conditions. The sky must be as clear as possible. In order to detect Cas $\mathrm{A}$, we should equip the antenna with a tracking system to observe the source during hours. We should also use a larger antenna together with a high sensitivity receiver to detect other radio sources.

\section{Conclusion}

Radio telescopes operating at decimetre and centimetre wavelengths may be built in developing countries where the weather conditions are not favourable for optical observations. The antennae and most of the electronic equipments can be bought at relatively low cost in the market, especially to build solar instruments. A solar radio interferometer was built to observe the 1995 solar eclipse in Vietnam. It was offered to the National University of Vietnam in Hanoi to train students in physics. This kind of instrument can be used to observe strong radio sources. Simple interferometers consisting of a pair of 5-m 
antennae can be built to observe sunspots, but the building technique does not require any special expertise.

\section{References}

de Bernardis, P., et al., 2000, Nature 404, 955.

Biraud, F., 1985, l'Astronomie, November Issue, p. 529.

Biraud, F., and Darchy, B., 1990, March, Report of Station de Radio Astronomie de Nancay.

Boulanger, F, Viallefond, F., 1992, Astron.Astrophys. 266, 37.

Darchy, B., and Flouret, B., 1999, January, Report of Station de Radio Astronomie de Nançay.

Hanany, S., et al., 2000, Astrophys. J. submitted.

Hulst, van de, H.C., Muller, C.A., and Oort, J.H., 1954. Bull. Astr. Netherlands, $12,117$.

Nguyen-Q-Rieu, 1993, in Microwave Engineering Handbook, vol.3, Ed. B. Smith and $\mathrm{M}-\mathrm{H}$. Carpentier (Chapman and Hall), p. 511.

Penzias, A.A., and Wilson, R.W., 1965, Astrophys. J. 142, 419.

Sancisi, R., and van Albada, T.S., 1987, in Dark Matter in the Universe (IAU Symp. Nr. 117), Eds. J Kormendy and G.R. Knapp, p. 67, D. Reidel, Dordrecht, The Netherlands.

Smoot, G.F. et al., 1991, Astrophys. J. 371, L1.

Thai-Q-Tung, 1997, Report, Observatoire de Paris.

\section{Discussion}

In answer to Fierro, who asked how many antennae had been built and are functioning, Rieu said that a few had been built at the Radio Astronomy Centre of the Paris Observatory and are functioning. As stated in the paper, one was used to observe the 1995 solar eclipse in Vietnam and it was offered to the National University of Hanoi, to train physics students. Wentzel observed that a small radio telescope had been built near Calcutta, as a student project in an engineering course supervised by Ramesh Sinha. Rieu remarked that, apart from its value for training physics students, such an instrument could be built by amateur astronomers. Schreuder stated that although radio astronomy is very important in The Netherlands, Dutch radio astronomers tended to think it too complicated for amateurs. Nevertheless, a group of Dutch amateurs, with professional guidance, had built and successfully operated an instrument very similar to the one described by Rieu. Rieu said that the group in question lived about $30 \mathrm{~km}$ from Westerbork and one of the examples he had shown came from Westerbork. Kochhar observed that local people living near the GMRT in Pune, India, felt their job opportunities restricted because of the opportunities created for radio astronomers. Rieu replied that this was a social problem for which Indian radio astronomers are not to blame. The GMRT is an excellent low-frequency interferometer. 